\title{
Feasibility of detection and intervention for alcohol-related liver disease in the community:
}

\author{
the Alcohol and Liver Disease Detection study (ALDDeS)
}

\begin{abstract}
Background

In the past 15 years mortality rates from liver disease have doubled in the UK. Brief alcohol advice is cost effective, but clinically meaningful reductions in alcohol consumption only occur in around 1 in 10 individuals.
\end{abstract}

\section{Aim}

To provide evidence that detecting early liver disease in the community is feasible, practical, and that feedback of liver risk can increase the proportion of subjects reducing alcohol consumption.

\section{Design and setting}

A community feasibility study in nine general practice sites in Hampshire.

\section{Method}

Hazardous and harmful drinkers were identified by WHO AUDIT questionnaire and offered screening for liver fibrosis.

\section{Results}

In total, 4630 individuals responded, of whom $1128(24 \%)$ hazardous or harmful drinkers were offered a liver fibrosis check using the Southampton Traffic Light (STL) test; 393 (38\%) attended and test results were returned by post. The STL has a low threshold for liver fibrosis with $45(11 \%)$ red, 157 (40\%) amber. and 191 (49\%) green results. Follow-up AUDIT data was obtained for 303/393 (77\%) and 76/153 $(50 \%)$ subjects with evidence of liver damage reduced drinking by at least one AUDIT category (harmful to hazardous, or hazardous to low risk) compared with 52/150 (35\%,

$P<0.011$ ) subjects without this evidence; in the subset of harmful drinkers patterns (AUDIT $>15), 22 / 34$ (65\%) of STL positives, reduced drinking compared with $10 / 29(35 \%, P<0.017)$ STL negatives.

\section{Conclusion}

Detection of liver disease in the community is feasible, and feedback of liver risk may reduce harmful drinking.

Keywords

alcohol; brief intervention; cirrhosis; liver prevention.

\section{INTRODUCTION}

Between 1970 and 2010 standardised mortality rates from diseases in all organs decreased in the UK, the single exception being liver disease, where death rates increased by more than $400 \%$. Of the 7501 recorded liver deaths in 2011 in England and Wales, $67 \%$ were under the age of 65 years, and according to the Office for National Statistics liver disease causes a greater loss of working years of life than lung cancer, chest disease, diabetes, or cerebrovascular disease.' Liver death rates in the UK have doubled over the past 15 years linked to increasing consumption of cheap alcohol bought from supermarkets and off licences and drunk at home.

The problem is that alcohol-related liver disease (ALD) develops silently, progressing from simple fatty liver through progressive fibrosis to cirrhosis and finally liver failure and death. Once patients are seen by specialist liver services half will stop drinking, but half of these patients die before their liver has a chance to recover with only the remaining half surviving long term. ${ }^{2}$

Of 4395 first admissions to Southampton General Hospital with cirrhosis or liver failure between 1996-2012, 3306 (75\%) had not been seen previously in a liver clinic ( $N$ Sheron, unpublished data, 2013).

And another study has found that GPs had discussed alcohol use in less than one-third

N Sheron, MD, FRCP, head of clinical hepatology; S Harris, BSc, MSc, statistician; P Roderick, MD, FRCP, FFPH, professor of public health, Faculty of Medicine, University of Southampton, Southampton. M Moore, MSc, MRCP, FRCGP, reader in primary care research, Primary Care Research Network South, Aldermoor Health Centre, Southampton. W O'Brien, RN, senior research sister, ALDDeS \& HEAT Trials, Primary Medical Group, Aldermoor Health Centre,

Southampton

\section{Address for correspondence}

Nick Sheron, University of Southampton Faculty of cases subsequently admitted to hospital with ALD. ${ }^{3}$ One-third of ALD admissions die within a year as a result of liver failure or variceal haemorrhage, never having had the opportunity to benefit from abstention, whereas the survival curves in abstainers who survive this initial period are flat after about 18 months. ${ }^{2}$ Patients are developing ALD in the community but it is not identified until they are admitted to hospital with endstage disease, by which time it is too late for many of them.

If high-risk drinking behaviour is identified in the community then brief advice or intervention is an effective and cost-effective means to reduce drinking; numerous randomised controlled trials have been summarised in systemic reviews, metaanalyses and NICE reports..$^{4-6}$ Numbers needed to treat are around 1 in $8-12$, so around $10 \%$ of patients reduce drinking by a significant degree. This contrasts with $50 \%$ of patients who stop drinking when informed by a liver specialist that they have alcohol related cirrhosis. ${ }^{2}$

All liver disease follows a common pathway of fibrosis, leading to cirrhosis and finally chronic liver failure, or more commonly acute on chronic liver failure. The study therefore formed the hypothesis that if there was a liver test which could be used to detect liver fibrosis at an early stage in the community, and this information was fed of Medicine, Clinical and Experimental Sciences Academic Unit, University Hospital Southampton NHS Foundation Trust, Tremona Road,

Southampton, S016 6YD.

E-mail: Nick.Sherondsoton.ac.uk

Submitted: 4 October 2012; Editor's response: 8 April 2013; final acceptance: 4 June 2013. (CBritish Journal of General Practice This is the full-length article (published online 30 Sep 2013) of an abridged version published in print. Cite this article as: Br J Gen Pract 2013; DOI: 10.3399/bjgp13X673711 


\section{How this fits in}

Community-based alcohol interventions generally have a number needed to treat of 8-12, but after a liver admission approximately half of the patients stop drinking. This study brings specialist non-invasive diagnostic technology into primary care to detect early stages of liver fibrosis with the specific aim of changing behaviour. The technology is intuitively simple and affordable, and stopped harmful drinking in $65 \%$ of patients with evidence of early liver disease.

back to patients, then we could potentially prevent more people from presenting far too late with the complications of alcohol related cirrhosis.

Patients with suspected liver disease are currently investigated using a panel of liver function tests, with the greatest importance paid to the levels of the liver enzyme alanine transaminase (ALT). An elevated ALT is related to the degree of fatty infiltration in the liver but has no discrimination for identifying subjects with progressive liver fibrosis or cirrhosis. ${ }^{7}$ In recent years a range of new liver diagnostic technologies have been introduced into secondary care which can detect the fibrosis directly, and the study hypothesised that these non-invasive tools could be used in primary care to detect early liver disease when risk reduction could prevent the development of serious liver disease. With this aim in mind the study developed the Southampton Traffic Light (STL) test, ${ }^{7}$ a relatively inexpensive (£50) blood test which combines two well-established markers of liver fibrosis, hyaluronic acid, and collagen P3NP. together with platelet count as a measure of portal hypertension. The study recently published follow-up data in more than 1000 subjects who had the STL, followed for up to 9 years, showing that categorisation of STL into three risk groups initially termed: strong positive/positive/negative had good prognostic accuracy. ${ }^{7}$ The grades were modified to red/amber/green traffic light results during the course of the study.

This study presents results from a community-based feasibility study to test out population screening of adults for alcohol misuse followed by assessment and feedback of the degree of liver injury using the STL in those drinking hazardously or harmfully, with one year follow-up to determine feasibility of follow-up and to examine if there were changes in alcohol use.

\section{METHOD}

\section{Subjects and setting}

Nine general practice sites across Hampshire, Wiltshire, and Dorset, which provided a mix of both urban and inner-city sites, were identified by the South West Primary Care Research Network. Potential participants were identified by first selecting those aged between 25-54 years from the practice list and then random sampling from this selection.

\section{Inclusion/exclusion criteria}

All adults aged 25-54 years were eligible for the study. The mean age of admission for alcohol-related liver disease in Southampton is 54 years; as the aim of this study was to pick up early disease, this age was used as the upper cut-off for the study. Those screened out by their GPs were excluded if a postal questionnaire was considered inappropriate, for instance, those receiving terminal care or those with severe mental health problems. Individuals previously known to have a history of alcohol-related problems, alcohol-related liver problems, or other liver disease were not excluded.

\section{Recruitment}

Each eligible participant was sent an invitation letter signed by their GP, along with an information sheet and questionnaire, which included the WHO AUDIT questionnaire, the gold standard reference for detecting harmful and hazardous drinking patterns. ${ }^{8}$ Those scoring $<8$ were thanked for their response but took no further part in the study. Those identified as drinking at hazardous levels (AUDIT $\geq 8$ ) were invited to attend a research nurse-led clinic. A single followup letter was sent to non-responders. At the screening visit participants were provided with study information and having completed written informed consent were entered into the study. Baseline data were collected, which included:

- a detailed alcohol history, including daily and weekly drinking patterns;

- sociodemographic data;

- relevant clinical history;

- a completed 20-item Severity of Alcohol Dependence Questionnaire (SADQ), a validated assessment tool for harmful drinking and alcohol dependency; ${ }^{9}$ and

- the patient's postcode of residence, which was used to derive the Index of Multiple Deprivation (IMD) (2007). ${ }^{10}$ 


\section{Liver assessment and STL}

Examination included height, weight, and waist circumference of each participant and a non-fasted venous sample was taken for serum ALT, gamma-glutamyltransferase, albumin, full blood count, clotting, and fibrosis markers (hyaluronic acid and P3NP). All blood tests were performed as routine tests in the NHS clinical biochemistry and haematology labs at University Hospital Southampton using assays as previously described?

All patients with an AUDIT score $\geq 8$ (those hazardous and harmful drinkers) were given written information, Alcohol and Sensible Drinking. ${ }^{11}$ This leaflet was used as the control intervention in the current trial of brief intervention by the National Brief Intervention Research Consortium recently funded by the Department of Health. ${ }^{12}$

The liver-check test results were communicated to patients as follows:

- Negative (green test): "We are pleased to say that, at the moment, there is no sign of any severe liver fibrosis in the blood test. We do recommend that you examine your alcohol intake and check that you are drinking at safe levels to prevent future liver damage'.

Figure 1. Study flowchart.

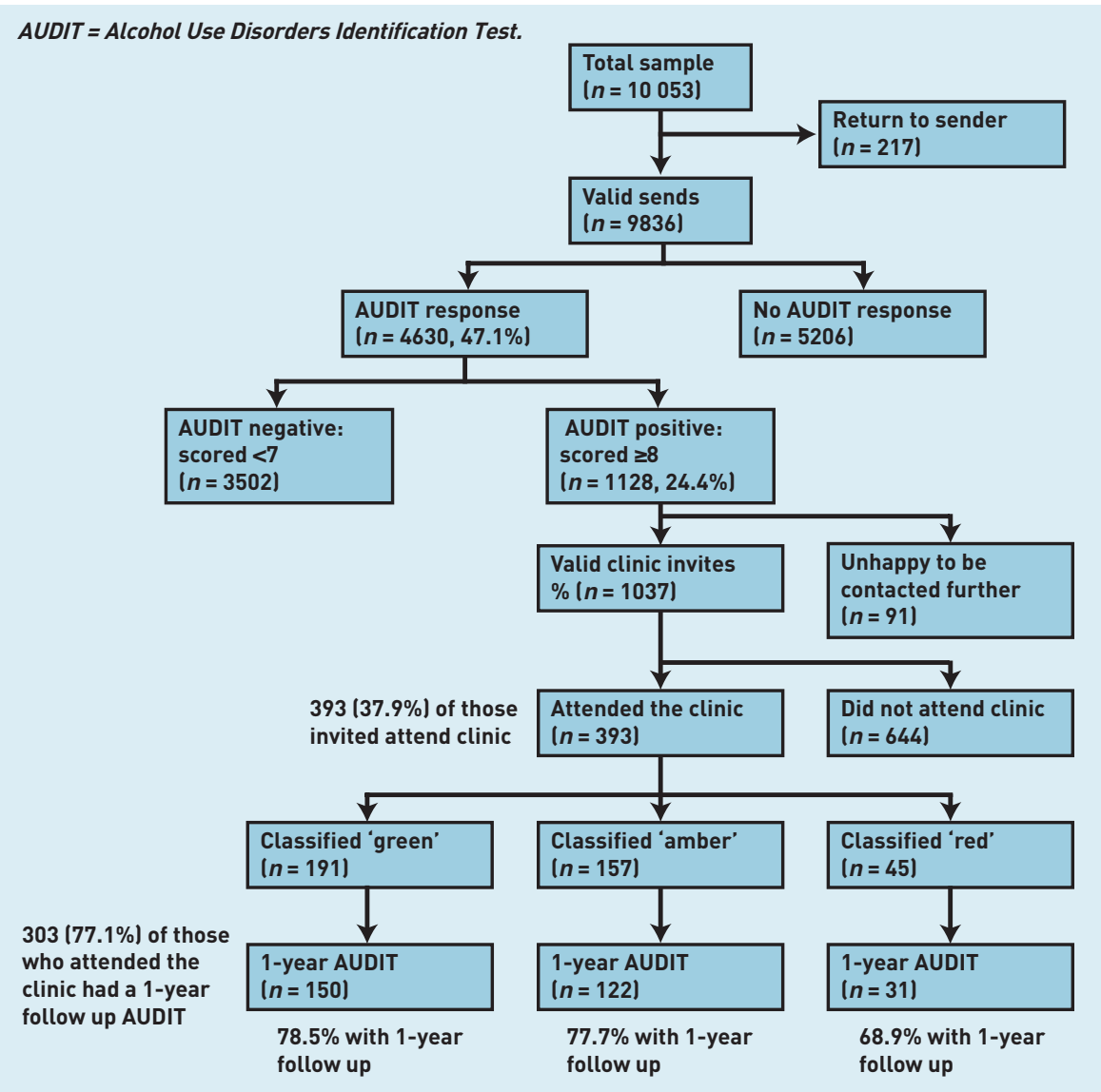

- Positive (red/amber test): 'We are writing to inform you that the test for liver fibrosis shows that your liver may be damaged. Further damage may be preventable by reducing your alcohol consumption to safe levels but we recommend that you have a further assessment of your liver health, which can be arranged by your own GP'.

The follow-up for patients was performed by their own GP who were given additional information to help interpret test results in a simple schema as follows:

- Negative/green: severe liver fibrosis unlikely, but early disease cannot be excluded;

- Positive/amber: some degree of liver fibrosis possible;

- Strong positive/red: liver fibrosis probable, with severe liver fibrosis or cirrhosis a possibility.

GPs were alerted to participants with evidence of moderate or severe alcohol dependency as judged by the AUDIT/SADQ so that an appropriate referral to specialist alcohol services could be made if clinically indicated. It was suggested to GPs that subjects with a red test were referred to a liver clinic and subjects with an amber test should undergo further assessment for liver disease either in the practice or via referral to a liver clinic. All decisions about further follow-up, investigation, or referral were performed by the patient's own practice.

All participants with an AUDIT score $\geq 8$ were invited to complete a follow-up AUDIT questionnaire 8-12 months following their initial response and given the opportunity to attend for a follow-up screening visit, regardless of whether they attended at baseline at which a repeat blood test for the STL was taken. The change in category of AUDIT score was determined as follows:

- low risk <8;

- hazardous 8-15; or

- harmful/dependent $\geq 16$.

\section{Statistical methods}

Descriptive statistics are presented separately for each of the traffic light categories. Frequencies and percentages are presented for the categorical variables and means with standard deviations for the continuous variables. Skewed variables also include median, lower, and upper quartiles.

Comparisons between the traffic light categories were made with $\chi^{2}, \chi^{2}$ trend, 
Fisher's exact, analysis of variance (ANOVA), and Kruskal-Wallis tests, as appropriate. Characteristics of those who returned for 1-year follow-up were compared with nonreturners using Pearson $\chi^{2}$. Fisher's exact, and Mann-Whitney $U$ tests, as appropriate. Comparisons of change within the traffic light categories were made with paired samples $t$-tests for the AUDIT score and $\chi^{2}$ tests for changes in AUDIT category.

Red and amber categories were merged as both highlighted liver risk in the feedback and because changes were very similar in these categories (data not shown). Both the completed case analysis and sensitivity analysis were undertaken using all cases and assuming missing cases did not change their baseline alcohol use. $P$-values of $<0.05$

\begin{tabular}{|c|c|c|c|c|}
\hline Variable & $\begin{array}{c}\text { Green, } \\
n=191 \\
n(\%)\end{array}$ & $\begin{array}{c}\text { Amber, } \\
n=157 \\
n(\%)\end{array}$ & $\begin{array}{c}\text { Red, } \\
n=45 \\
n(\%)\end{array}$ & $P$-value \\
\hline \multicolumn{5}{|l|}{ Age, years } \\
\hline Median & 43 & 45 & 45.5 & \multirow[t]{3}{*}{$0.020^{\mathrm{a}}$} \\
\hline Lower to upper quartile & $37-48$ & $39-51$ & $34-52$ & \\
\hline Minimum-maximum & $25-55$ & $25-54$ & $26-55$ & \\
\hline \multicolumn{5}{|l|}{ Sex } \\
\hline Male & $95(50)$ & $101(64)$ & $33(73)$ & $0.002^{b}$ \\
\hline \multicolumn{5}{|c|}{ Deprivation (IMD score: May 2009) } \\
\hline Quintile 4 or 5 & $66(40)$ & $51(40 \%)$ & $12(29)$ & $0.421^{b}$ \\
\hline \multicolumn{5}{|l|}{ Ethnicity } \\
\hline Non white & $14(7.6)$ & $14(9.2)$ & $3(6.8)$ & $0.863^{c}$ \\
\hline \multicolumn{5}{|l|}{ Marital status } \\
\hline Single & $46(24)$ & $21(14)$ & $11(25)$ & \multirow[t]{3}{*}{$0.003^{c}$} \\
\hline Married/cohabiting & 137 (73) & $112(74)$ & $30(68)$ & \\
\hline Separated/divorced & $6(3.2)$ & $19(13)$ & $3(6.8)$ & \\
\hline \multicolumn{5}{|l|}{ AUDIT score } \\
\hline Median & 11 & 11 & 12 & \multirow[t]{3}{*}{$0.499^{a}$} \\
\hline Lower to upper quartile & $9-15$ & $9-15$ & $9.5-15.5$ & \\
\hline Minimum-maximum & $8-36$ & $8-33$ & $8-36$ & \\
\hline$>15$ & $42(22)$ & $35(22)$ & $11(24)$ & $0.938^{b}$ \\
\hline \multicolumn{5}{|l|}{ SADQ score } \\
\hline$>15$ & $14(7.4)$ & $4(2.6)$ & $7(16)$ & $0.005^{c}$ \\
\hline \multicolumn{5}{|l|}{ ALT/SGPT (iu/L) } \\
\hline \multirow{2}{*}{\multicolumn{5}{|c|}{$\begin{array}{l}>\text { ULN (40: male, } \\
35: \text { female) }\end{array}$}} \\
\hline & $21(11)$ & $15(10)$ & $11[26]$ & $0.019^{\circ}$ \\
\hline \multicolumn{5}{|l|}{ Total units/week } \\
\hline Median & 25.5 & 26.8 & 36 & \multirow[t]{3}{*}{$0.012^{\mathrm{a}}$} \\
\hline Lower to upper quartile & $16-43$ & $18-38.4$ & $23-64.5$ & \\
\hline Minimum-maximum & $0-189$ & $3-113.8$ & $4-210$ & \\
\hline \multicolumn{5}{|l|}{ Drinking days/week } \\
\hline Median & 4 & 4 & 4 & \multirow[t]{3}{*}{$0.208^{a}$} \\
\hline Lower to upper quartile & $3-6$ & $2.3-5$ & $3-6$ & \\
\hline Minimum-maximum & $0-7$ & $0-7$ & $1-7$ & \\
\hline
\end{tabular}

${ }^{a}$ Kruskal-Wallis test. ${ }^{b}$ Pearson $\chi{ }^{2} .{ }^{c}$ Fisher's exact test. $A L T=$ alanine transaminase. $A U D I T=A l c o h o l ~ U s e$ Disorders Identification Test. IMD = Index of Multiple Deprivation. SADQ = Severity of Alcohol Dependence Questionnaire. SGPT = serum glutamic-pyruvic transaminase. ULN = upper limit of normal. were considered statistically significant. All analyses were performed using IBM SPSS Statistics (version 19).

\section{RESULTS}

In total, 9836 valid AUDIT questionnaires were distributed; 4630 (47\%) completed questionnaires were returned (Figure 1). Response rates between general practices varied from 25-66\%. Responses using anonymised data provided by sites were compared: responders were significantly more likely to be older, female, and to have lower deprivation scores. Of those who did respond, those who were younger, male, and more deprived were more likely to be harmful/dependent drinkers with an AUDIT score of $>14$. Of the responders, 1128 (24\%) had an AUDIT score of $\geq 8$ and of these 1037 $(92 \%)$ indicated that they were prepared to join the study (Figure 1).

All those AUDIT positive who were willing to continue in the study were invited to attend a nurse-led, liver screening clinic at their own surgery, but only 393/1037 (38\%) attended. Of these attenders, 305 (78\%) were hazardous drinkers with an AUDIT score of $8-15$, and $88(23 \%)$ were harmful/dependent drinkers with an AUDIT score of more than 15. Characteristics of those attending the assessment clinic are shown in Table 1 by traffic light category. There were 45 (11\%) red 157 (40\%) amber and 191 (49\%) green. This clinic population as a whole was not characterised by extreme drinking, with a median reported alcohol consumption of 27 units per week (interquartile range [IQR] 18-42); the harmful/dependent subgroup had higher alcohol consumption: median 43 units/week (IQR 27-69). Factors associated with higher STL risk were older age, being male, not being married, alcohol consumption lunits per week though not days drinkingl and dependency on SADQ, though not the AUDIT score.

\section{Follow-up AUDIT}

Follow-up AUDIT questionnaires were obtained from 303/393 clinic attenders (77\%) after a median follow-up of 370 days (IQR 330-427 days, Figure 1). There were no differences in response by age, sex, and drinking days, or by traffic light category, but non-responders had significantly higher baseline AUDIT scores and alcohol consumption.

On an intention-to-treat basis, assuming those who did not return for follow-up were unchanged, the group overall reduced its AUDIT score from 13 to 11 (in 393) and $133 / 393(34 \%)$ reduced by one grade or more. 
Table 2. Baseline and follow-up AUDIT scores for those attending the clinic, by traffic-light category

\begin{tabular}{|c|c|c|c|c|}
\hline Variable & $\begin{array}{l}\text { Green, } \\
n=191\end{array}$ & $\begin{array}{c}\text { Amber/red, } \\
n=202\end{array}$ & $\begin{array}{c}\text { Total } \\
n=393\end{array}$ & $P$-value \\
\hline Response to 1-year AUDIT, n (\%) & $150(79)$ & $153(76)$ & 303 (77) & \\
\hline \multicolumn{5}{|l|}{ Baseline AUDIT } \\
\hline Mean (SD) & $13.0(5.7)$ & $12.8(5.1)$ & $12.9(5.4)$ & $0.893^{a}$ \\
\hline Median & 11 & 11 & 11 & \\
\hline Lower to upper quartile & $9-15$ & $9-15$ & $9-15$ & \\
\hline Minimum-maximum & $8-36$ & $8-36$ & $8-36$ & \\
\hline \multicolumn{5}{|l|}{ 1-year follow-up AUDIT } \\
\hline Mean (SD) & $10.6(5.4)$ & 9.5 (5.3) & $10.0(5.4)$ & $0.070^{a}$ \\
\hline Median & 9 & 9 & 9 & \\
\hline Lower to upper quartile & $7-12.3$ & $6-12$ & $6-12$ & \\
\hline Minimum-maximum & $3-29$ & $0-36$ & $0-36$ & \\
\hline \multicolumn{5}{|c|}{ Change in AUDIT at follow-up (negative value is a reduction) } \\
\hline Mean (SD) & $-1.9(3.6)$ & $-3.0(4.5)$ & $-2.4(4.1)$ & $0.014^{b}$ \\
\hline Minimum-maximum & -15 to 7 & -17 to 9 & -17 to 9 & \\
\hline \multicolumn{5}{|l|}{ Completed cases only } \\
\hline$P$-value for change & $<0.001^{c}$ & $<0.001^{c}$ & $<0.001^{c}$ & \\
\hline
\end{tabular}

${ }^{a}$ Mann-Whitney test. ${ }^{b}$ Independent samples $t$-test. ${ }^{c}$ Paired-samples t-test. AUDIT = Alcohol Use Disorders Identification Test. SD = standard deviation

AUDIT scores for the responders ( $n=303)$ are given in Table 2. The overall individual change in AUDIT score over time was a

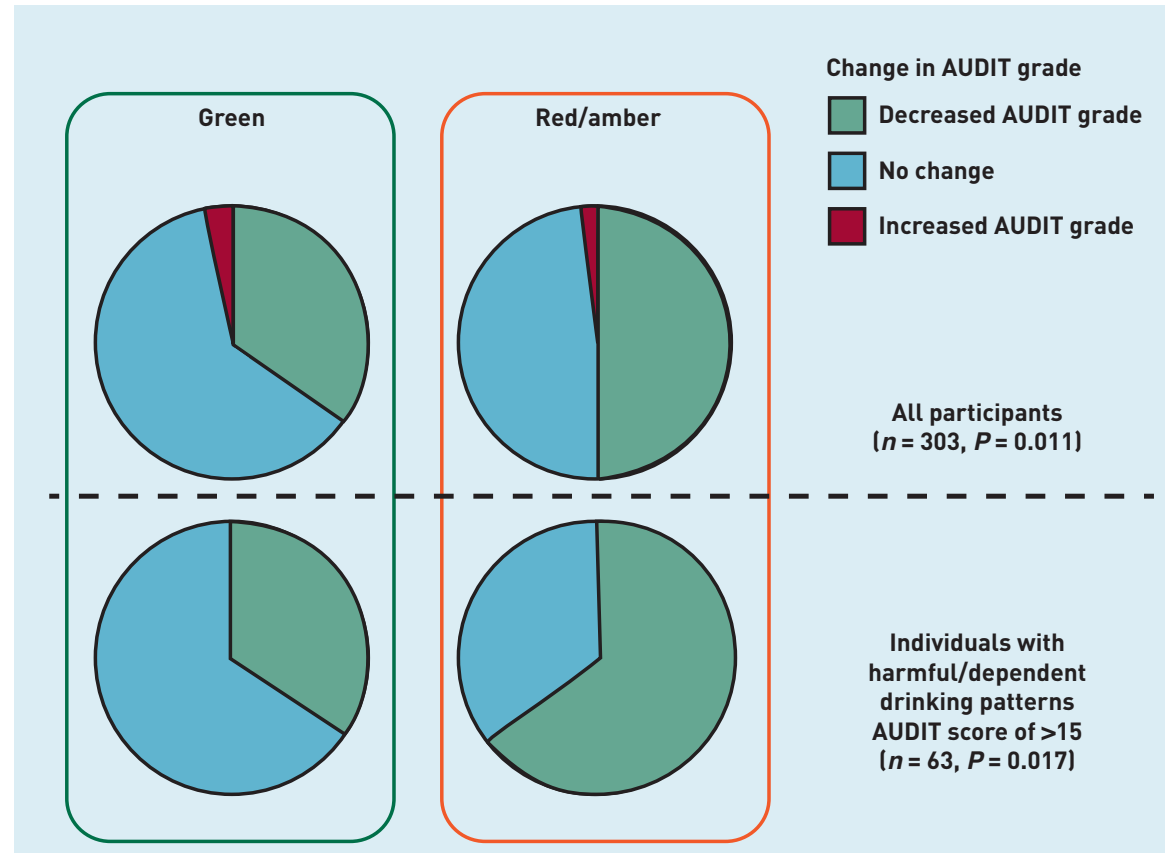

1-year follow-up was obtained in $303 / 393$ (77\%) subjects, of whom $240 / 303$ (79\%) were hazardous drinkers (AUDIT $\geq 8$ ) and 63/303 (21\%) harmful/dependent drinkers. Subjects given a positive (red/amber) test result were more likely to reduce their drinking (50\% versus $35 \%, \mathrm{P}=$ $0.011)$ by at least one AUDIT category, with the highest levels of behaviour change seen in very heavy drinkers (65\% versus 35\%, P = 0.017). AUDIT = Alcohol Use Disorders Identification Test.

differences in the AUDIT scores between STL grades at baseline but 1-year changes within individuals were significantly greater in the red/amber group (Table 2). The magnitude of effect was reduced, but the implications unchanged if non-responders were included and the assumed AUDIT score was unchanged.

Of the 303 responders, 128 (42\%) had reduced their drinking by at least one AUDIT grade, from harmful/dependent to hazardous, or from hazardous to low risk; $8(2.6 \%)$ individuals had an increased AUDIT grade. People given a positive test result (red/amber) were significantly more likely to reduce by one grade or more than the green group ( $P=0.011$, Table 3, Figure 2). In the subgroup of heavier drinkers with an original AUDIT >15 and a follow-up AUDIT response $(n=63)$ both the change in AUDIT score and percentages reducing by at least one AUDIT grade were significantly higher in the red/amber group compared with the green group ( $P=0.017$, Table 3, Figure 2).

\section{DISCUSSION}

\section{Summary}

The specialist management of alcoholic liver disease (ALD) is straightforward and has two components; to keep patients alive long enough for them to stop drinking, and to maximise the number of patients who stop drinking. If drinking behaviour could be changed in primary care, secondary care would become unnecessary. But whereas half of patients stop drinking when diagnosed in hospital with ALD $^{2}$ alcohol interventions in primary care are less successful. ${ }^{5}$ The purpose of the study was to examine the feasibility of bringing secondary care diagnostic technology care into primary care to change drinking behaviour, before it is too late.

The study has demonstrated the feasibility of postal screening of the adult primary care population for alcohol misuse and subsequent liver health assessment of a proportion of those identified at risk. All those responding with a positive AUDIT score received an alcohol advice leaflet. A total of 393 hazardous and harmful/dependent drinkers underwent the traffic light test, of whom 45 (12\%) had a red, and 157 (40\%) an amber traffic light. It is important to note that the standard 'Liver function test' panel had no discriminatory value for fibrosis as assessed by the STL test. In the liver clinic population in whom the test was developed, a red STL grade had a positive predictive value (PPV) for liver fibrosis of $96 \%$ and for cirrhosis of $69 \%$, the lower prevalence of liver fibrosis in the community would reduce 


\begin{tabular}{|c|c|c|c|c|}
\hline Variable & $\begin{array}{c}\text { Green, } \\
\begin{array}{c}n=191 \\
n(\%)\end{array}\end{array}$ & $\begin{array}{c}\text { Amber/red, } \\
\begin{array}{c}n=202 \\
n(\%)\end{array}\end{array}$ & $\begin{array}{c}\text { Total, } \\
n=393 \\
n(\%)\end{array}$ & $P$-value \\
\hline \multicolumn{5}{|l|}{ Baseline AUDIT category } \\
\hline$<8$ & $0(0)$ & $0(0)$ & $0(0)$ & $0.853^{a}$ \\
\hline $8-15$ & 149 (78) & $156(77)$ & 305 (78) & \\
\hline$>15$ & $42(22)$ & $46(23)$ & $88(22)$ & \\
\hline \multicolumn{5}{|l|}{ 1-year follow-up AUDIT category } \\
\hline$<8$ & $42(28)$ & 60 (39) & 102 (34) & $0.020^{\mathrm{a}}$ \\
\hline $8-15$ & $84(56)$ & $78(51)$ & 162 (53) & \\
\hline$>15$ & $24(16)$ & $15(10)$ & 39 (13) & \\
\hline \multicolumn{5}{|c|}{ Change in AUDIT category at 1 -year follow-up ( $n=303)$} \\
\hline Reduced by at least one level & 52 (35) & $76(50)$ & 128 (42) & $0.011^{a}$ \\
\hline No change & $93(62)$ & $74(48)$ & $167(55)$ & \\
\hline Increased by at least one level & 5 (3.3) & $3(2.0)$ & $8(2.6)$ & \\
\hline \multicolumn{5}{|c|}{$\begin{array}{l}\text { Change in AUDIT category in subset of those with harmful/dependent drinking patterns } \\
\text { (initial AUDIT }>15, n=63 \text { ) }\end{array}$} \\
\hline Reduced by at least one level & $10(35)$ & 22 (65) & $32(51)$ & $0.017^{\mathrm{a}}$ \\
\hline No change & $19(66)$ & 12 (35) & $31(49)$ & \\
\hline
\end{tabular}

\section{Funding}

This paper presents independent research funded by the National Institute for Health Research (NIHR) under its Research for Patient Benefit (RfPB) Programme (Grant Reference number: PB-PG-0906-11080). The views expressed are those of the authors and not necessarily those of the NHS, NIHR, or the Department of Health.

\section{Ethical approval}

The study was approved by the Southampton and South West Hampshire Research Ethics Committee A, 07/H0502/160.

\section{Provenance}

Freely submitted; externally peer reviewed.

\section{Competing interests}

The authors have declared no competing interests.

\section{Additional information}

An online calculator for the Southampton Liver Traffic Light test is available at: http:// livertrafficlight.info/

\section{Discuss this article}

Contribute and read comments about this article on the Discussion Forum: http://www.rcgp.org.uk/bjgp-discuss the PPV somewhat but the study estimates that between one-half and three-quarters of these 'red' individuals have a significant degree of liver fibrosis.?

The study obtained a follow-up AUDIT in $77 \%$ of those attending for traffic light tests, of whom $42 \%$ reduced their drinking by at least one AUDIT hazard grade, either from hazardous (AUDIT 8-15) to low risk, or from harmful/dependent (AUDIT >15) to hazardous. Although it is unlikely that subjects who failed to return the follow-up AUDIT were all 'intervention fails', in this sensitivity analysis of all cases the proportion of patients cutting alcohol reduced from $42 \%$ to $33 \%$. A reduction of at least one AUDIT grade was significantly more likely to occur in subjects given a red/amber STL $(50 \%)$ compared with those given a green STL (35\%).

The harmful/dependent drinkers in the study drank a mean of 53 units $(1$ unit = $1 \mathrm{cl}$ of pure alcoholl each week compared with 28 units for hazardous drinkers. It is a common misconception that heavier drinkers are less likely to respond to health interventions, for example meta-analyses of brief interventions often exclude patients with harmful/dependent drinking patterns. ${ }^{13}$ Whereas the study's clinical experience is that many of these patients are highly responsive to appropriate behavioural advice in the context of serious liver disease, ${ }^{2}$ which is also consistent with evidence from another primary care study in which readiness change was greater in those with greater severity of alcohol misuse. ${ }^{14}$ In this study's subset of harmful/dependent drinkers it was found that $65 \%$ of individuals with a red/amber result reduced drinking by one AUDIT grade, nearly twice as many as those with green STL (35\%). On an intention to treat analysis drinking was reduced to safe levels in $48 \%$ of harmful drinkers with any evidence of liver fibrosis as evidenced by a red/amber traffic light, the same degree of behaviour change seen in patients with alcohol-related cirrhosis; but if we wait for patients to present with liver failure or variceal haemorrhage it is too late for many of them to benefit.

\section{Strengths and limitations}

There were two components to this study:

- the screening and recruitment of subjects up to the point when they underwent the STL test; and

- the impact of this early liver diagnosis on their behaviour.

Over the course of a few months a single work time equivalent research nurse completed a postal screening survey for unhealthy alcohol consumption in nearly 10000 subjects in the community of whom $47 \%$ responded. In terms of the proportion of subjects attending for the liver check-up, the attrition rate was higher than initially expected. An attempt was made to modify the protocol to be more responsive to patients but with no increase in response (data not shown). Those attending for the liver check may have been more likely to change drinking behaviour. The study was concerned that subjects with a negative liver test may see this as a 'licence to drink', and clearly alcohol reduction provides health benefits in hazardous and harmful drinkers irrespective of liver status. So the study was careful to emphasise to GPs who fed back the results to patients that a green traffic light result did not exclude early fibrosis. This question is addressed in more detail in the qualitative study which was conducted alongside the main study (reported separately). ${ }^{15}$ Given that all subjects drinking at hazardous or harmful levels are at increased risk of a range of medical consequences, for future studies in patients the categories were rephrased for feedback for these patients, so green becomes amber, amber becomes red, and red maroon or black. This will reduce the possibility of false re-assurance in hazardous/harmful drinkers who have a green test.

Response rates varied between practices 
and were lower in practices with higher levels of deprivation, in which liver disease mortality is higher ${ }^{16}$ so it should not be assumed that this approach is generalisable to all areas of the UK.

A relatively high proportion of subjects reduced their drinking in comparison to previous studies of brief interventions, and it is not clear if this is due to the nature of the intervention; a specific test for liver disease, or because the study selected subjects more likely to respond. It is not known whether subjects failed to attend for the STL having completed the initial AUDIT screen because they had no intention of changing their behaviour, or because they had already cut their drinking. This selection bias is a necessary feature of all studies of alcohol interventions; were the study to make the unlikely assumption that everyone who chose not to take part in the study did so because they had no intention of cutting their alcohol intake then only $11 \%$ of AUDIT positive subjects would have improved drinking by one AUDIT grade.

Irrespective of the analysis, nearly twice as many harmful/dependent drinkers with evidence of liver fibrosis reduced their drinking compared with individuals with a green test result. This suggests that liver health feedback may be a useful adjunct for behaviour change but these results should be confirmed in a randomised controlled trial.

\section{Comparison with existing literature}

The many randomised controlled studies of brief intervention have shown a reduction in drinking in the intervention arm amounting to $4-5 / 30 \mathrm{cl}$ (UK units) per week, or around $15 \%$ in comparison to the controls, ${ }_{17}^{17}$ with a number needed to treat (NNT) generally quoted of 1:8-12.18-19 In many of these studies reductions in drinking ranging from $12-46 \%$ were seen in the control arm as well. ${ }^{20}$ As a preliminary investigation of principle the study cannot be really be compared to these randomised interventions, but the overall levels of behaviour change are not dissimilar. Although the attrition rate was relatively high in the study, with subjects dropping out at various points in the screening process, this is consistent with previous studies of alcohol interventions where attrition rates of 44-83\% have been noted. ${ }^{21}$ This does have significant implications for the effectiveness of the postal screening approach that was used, the advantages being that it is cheap, quick and easy to implement, at the cost of missing many subjects.

A systematic review of 'tailored' or targeted health messages found a small but significant benefit in this approach ${ }^{22}$ which may be condition specific, for instance, there is little evidence to support feedback of DNA -based risk ${ }^{23}$ or tailored cardiovascular risk ${ }^{24}$ is effective for behaviour change whereas feedback of lung age (irrespective or risk) improved quit rates. ${ }^{25-27}$

\section{Implications for practice}

It has been shown that a reasonable proportion of subjects in the community will respond positively to an enquiry about their drinking and the offer of a liver health check, and that the results of this health check are encouraging in that feedback of liver risk appeared to influence their subsequent drinking behaviour, with the biggest reductions in drinking seen in the highest risk groups. The STL screening test may have other benefits, it identifies subjects at the highest risk of liver disease who can be referred for more intensive treatment and follow-up, and just as importantly it identifies a group with very low likelihood risk of liver fibrosis, potentially reducing unnecessary secondary care liver referrals. The 'medicalisation' of the process of liver diagnosis and alcohol intervention may help increase the uptake of this modality in primary care with the potential to reduce healthcare costs overall via reduced admissions and more selective clinic referrals.

The study has demonstrated the feasibility of postal screening and liver health check in primary care with promising results on behaviour change, and the results should be tested in a randomised controlled trial with economic analysis. The simple STL test used was intuitively understood by patients and GPs with a red test giving a high likelihood of significant underlying liver disease. An amber test is less specific for liver fibrosis but this is not the point, because providing the patient reduces their drinking to safe levels then ALD is not going to progress and the exact degree of liver damage is less relevant. The treatment for ALD is to stop drinking, and giving GPs and patients better tools to diagnose early liver disease may eventually help to turn the tide of increasing deaths in the UK. 


\section{REFERENCES}

1. Office for National Statistics. Mortality statistics: deaths registered in England and Wales (Series DR), 2010. Newport, South Wales: Office for National Statistics, 2011.

2. Verrill C, Markham H, Templeton A, et al. Alcohol-related cirrhosis -early abstinence is a key factor in prognosis, even in the most severe cases. Addiction 2009; 104(5): 768-774.

3. Verrill C, Smith S, Sheron N. Are the opportunities to prevent alcohol related liver deaths in the UK in primary or secondary care? A retrospective clinical review and prospective interview study. Subst Abuse Treat Prev Policy 2006; 1: 16.

4. McCambridge J, Kypri K. Can simply answering research questions change behaviour? Systematic review and meta analyses of brief alcohol intervention trials. PLoS One 2011; 6(10): e23748.

5. Kaner EF, Dickinson HO, Beyer F, et al. The effectiveness of brief alcohol interventions in primary care settings: a systematic review. Drug Alcohol Rev 2009; 28(3): 301-323.

4. McCambridge J, Kypri K. Can simply answering research questions change behaviour? Systematic review and meta analyses of brief alcohol intervention trials. PLoS One 2011; 6(10): e23748.

6. National Institute for Clinical Guidance. PH 24 Alcohol-use disorders preventing the development of hazardous and harmful drinking. London: NICE, 2010.

7. Sheron N, Moore M, Ansett S, et al. Developing a 'traffic light' test with potential for rational early diagnosis of liver fibrosis and cirrhosis in the community. $\mathrm{Br} J$ Gen Pract 2012; DOI: 10.3399/bjgp12X654588.

8. Saunders JB, Aasland OG, Babor TF, et al. Development of the Alcohol Use Disorders Identification Test (AUDIT): WHO Collaborative Project on Early Detection of Persons with Harmful Alcohol Consumption--II. Addiction 1993; 88(6): 791-804.

9. Stockwell T, Murphy D, Hodgson R. The severity of alcohol dependence questionnaire: its use, reliability and validity. Br J Addict 1983; 78(2): 145-155.

10. Jordan H, Roderick P, Martin D. The Index of Multiple Deprivation 2000 and accessibility effects on health. J Epidemiol Community Health 2004; 58(3): 250-257.

11. Kenny T. Alcohol and sensible drinking. http://www.patient.co.uk/pdf/4191.pdf (accessed 19 Aug 2013).

12. Kaner $\mathrm{E}, \mathrm{Bland} \mathrm{M}, \mathrm{Cassidy} \mathrm{P}$, et al. Effectiveness of screening and brief alcohol intervention in primary care (SIPS trial): pragmatic cluster randomised controlled trial. BMJ 2013; 346: e8501.

13. Ballesteros J, Duffy JC, Querejeta I, et al. Efficacy of brief interventions for hazardous drinkers in primary care: systematic review and meta-analyses. Alcohol Clin Exp Res 2004; 28(4): 608-618.

14. Williams EC, Kivlahan DR, Saitz R, et al. Readiness to change in primary care patients who screened positive for alcohol misuse. Ann Fam Med 2006; 4(3): 213-220.

15. Eyles C, Moore M, Sheron N, et al. Acceptibility of screening for early detection of liver diease in hazardous/harmful drinkers in primary care. Br J Gen Pract 2013; 63(613):516-522.

16. Siegler V, Al-Hamad A, Johnson B, et al. Social inequalities in alcohol-related adult mortality by National Statistics Socio-economic Classification, England and Wales, 2001-03. Health Stat Q2011; (50): 4-39.

17. Kaner EF, Beyer F, Dickinson HO, et al. Effectiveness of brief alcohol interventions in primary care populations. Cochrane Database Syst Rev2007; (2): CD004148.

18. Ballesteros J, Gonzalez-Pinto A, Querejeta I, Arino J. Brief interventions for hazardous drinkers delivered in primary care are equally effective in men and women. Addiction 2004; 99(1): 103-108.

19. Kaner EF, Heather N. Brief interventions against excessive alcohol consumption Oxford textbook of medicine. Oxford: Oxford University Press, 2010: 1334-1336.

20. Jenkins RJ, McAlaney J, McCambridge J. Change over time in alcohol consumption in control groups in brief intervention studies: systematic review and meta-regression study. Drug Alcohol Depend 2009; 100(1-2): 107-114.

21. Edwards AG, Rollnick S. Outcome studies of brief alcohol intervention in general practice: the problem of lost subjects. Addiction 1997; 92(12): 1699-1704.

22. Noar SM, Benac CN, Harris MS. Does tailoring matter? Meta-analytic review of tailored print health behavior change interventions. Psychol Bull 2007; 133(4): 673-693.

23. Marteau TM, French DP, Griffin SJ, et al. Effects of communicating DNA-based disease risk estimates on risk-reducing behaviours. Cochrane Database Syst Rev 2010; (10): CD007275

24. Price HC, Griffin SJ, Holman RR. Impact of personalized cardiovascular disease risk estimates on physical activity-a randomized controlled trial. Diabet Med 2011; 28(3): 363-372

25. Parkes G, Greenhalgh T, Griffin M, Dent R. Effect on smoking quit rate of telling patients their lung age: the Step2quit randomised controlled trial. BMJ 2008; 336(7644): 598-600.

26. McClure JB. Are biomarkers a useful aid in smoking cessation? A review and analysis of the literature. Behav Med 2001; 27(1): 37-47.

27. Bize R, Burnand B, Mueller $Y$, Cornuz J. Biomedical risk assessment as an aid for smoking cessation. Cochrane Database Syst Rev 2005; (4): CD004705. 